\title{
Oriented Mixed Area and Discrete Minimal Surfaces
}

\author{
Christian Müller • Johannes Wallner
}

Received: 29 February 2008 / Revised: 25 May 2009 / Accepted: 25 May 2009 /

Published online: 7 July 2009

(C) Springer Science+Business Media, LLC 2009

\begin{abstract}
Recently a curvature theory for polyhedral surfaces has been established, which associates with each face a mean curvature value computed from areas and mixed areas of that face and its corresponding Gauss image face. Therefore a study of minimal surfaces requires studying pairs of polygons with vanishing mixed area. We show that the mixed area of two edgewise parallel polygons equals the mixed area of a derived polygon pair which has only the half number of vertices. Thus we are able to recursively characterize vanishing mixed area for hexagons and other $n$-gons in an incidence-geometric way. We use these geometric results for the construction of discrete minimal surfaces and a study of equilibrium forces in their edges, especially those with the combinatorics of a hexagonal mesh.
\end{abstract}

Keywords Oriented mixed area - Discrete curvatures - Geometric configurations · Discrete minimal surfaces $\cdot$ Reciprocal parallelity

\section{Introduction}

Minimal surfaces represent a prominent topic which has attracted great interest for a long time and which several times has been the object of significant new developments. They combine differential geometry with other fields, notably complex analysis. Also the recent field of discrete differential geometry has not neglected them, and indeed the present paper represents a contribution to this area. Before entering into details, we want to say a few general words on the analogies and differences between the smooth and discrete categories.

C. Müller · J. Wallner (凶)

Institute of Geometry, TU Graz, Kopernikusgasse 24, 8010 Graz, Austria

e-mail: j.wallner@tugraz.at

C. Müller

e-mail: christian.mueller@tugraz.at 
The appeal of smooth minimal surface theory is to a large extent due to the fact that the same class of surfaces is characterized by different properties which are unrelated a priori, such as vanishing mean curvature, local surface minimization, or analyticity of isothermic parameterizations. Accordingly there is a variety of constructions of minimal surfaces: as solutions of Plateau's problem, as real part of Lie's sum of curves surfaces, or by Christoffel duality.

Transferring all these properties to the discrete category at the same time is not easy. Obviously we can pick a class of discrete surfaces (for instance, triangle meshes) and consider those discrete surfaces which enjoy a certain property analogous to one of the known properties of smooth minimal surfaces. However it is not guaranteed that these discrete minimal surfaces have any of the other properties which make their smooth counterparts such an interesting object of study. Nevertheless, for some appropriate discretizations, this is exactly what happens, and it is a major aim in discrete differential geometry to find them.

The following constructions stand out: Pinkall and Polthier [5] considered the class of triangle meshes and defined minimality by surface minimization. The resulting discrete minimal surfaces are, among others, minimizers of Dirichlet energy, capable of discrete conjugate surfaces, and allow for the solution of a discrete Plateau's problem. Bobenko and Pinkall [1] studied discrete isothermic surface parameterizations (this means quadrilateral meshes with planar faces and a cross ratio condition for the vertices). This approach led to a discrete Christoffel duality for isothermic parameterizations, where minimal surfaces and spheres correspond to each other, just as in the smooth case. This viewpoint is assumed by several papers based on [1], e.g., [10]. Bobenko, Hoffmann, and Springborn [4] took the idea of Christoffel duality further and applied it to Koebe polyhedra. They constructed a circle-based class of discrete minimal surfaces which exhibits convergence to the smooth case and makes it possible to find minimal surfaces from the combinatorics of the network of principal curvature lines.

It turned out that the discrete curvature theory for polyhedral surfaces introduced by Pottmann et al. [6], which is based on the variation of surface area in offset surfaces, contains both [1] and [4] as special cases. Minimality of a polyhedral surface with respect to an edgewise parallel Gauss image is in that context defined by vanishing mixed area of corresponding faces.

This brings us to the topic of the present paper, which systematically studies the vanishing mixed area property for polygons, having in mind as a main application the discrete minimal surfaces in the class of hexagonal meshes. After setting up the necessary definitions in the rest of this section, we continue with a geometric recursion for the computation of oriented mixed areas in Sect. 2. In Sect. 3 this leads to ways of characterizing pairs of parallel polygons whose mixed area is zero. Section 4 considers hexagonal meshes which are minimal and uses the incidence-geometric characterizations of vanishing mixed area which were obtained earlier for the construction of equilibrium forces in the edges of a minimal mesh. 


\subsection{Convex Polygons and Parallelity}

For two convex subsets $K, L \subseteq \mathbb{R}^{2}$, the area of nonnegative Minkowski combinations $\lambda K+\mu L$ obeys the law

$$
\operatorname{area}(\lambda K+\mu L)=\lambda^{2} \operatorname{area}(K)+2 \lambda \mu \operatorname{area}(K, L)+\mu^{2} \operatorname{area}(L),
$$

where the symbol area $(K, L)$ means the mixed area of $K$ and $L$ (see, for example, [9]). If the boundary $\partial K$ is a polygon $P$ with vertices $p_{0}, \ldots, p_{N-1}$, then the oriented area of $K$ is given by Leibniz' sector formula

$$
\operatorname{area}(P):=\operatorname{area}(K)=\frac{1}{2} \sum_{0 \leq i<N} \operatorname{det}\left(p_{i}, p_{i+1}\right) .
$$

Here indices are taken modulo $N$. Obviously, there is a vector space of polygons with $N$ vertices, and the area functional (2) is a quadratic form in this space. For us, the most interesting case is that $K$ and $L$ are bounded by parallel polygons $P$ and $Q$ with vertices $p_{0}, \ldots, p_{N-1}$ and $q_{0}, \ldots, q_{N-1}$, respectively. This concept, which is not restricted to convex polygons, was introduced by [6] and means that

$$
p_{i+1}-p_{i}, q_{i+1}-q_{i} \text { are linearly dependent for } i=0, \ldots, N-1 \text {. }
$$

We assume for a moment that both $P, Q$ have only nonzero edges (i.e., no coinciding vertices). Then the boundary $\partial(\lambda K+\mu L)$ has the vertices $\left(\lambda p_{i}+\mu q_{i}\right)_{0 \leq i<N}$, whence $\operatorname{area}(\lambda K+\mu L)=\frac{1}{2} \sum_{0 \leq i<N} \operatorname{det}\left(\lambda p_{i}+\mu q_{i}, \lambda p_{i+1}+\mu q_{i+1}\right)$, and consequently

$$
\operatorname{area}(P, Q)=\frac{1}{4} \sum_{0 \leq i<N}\left(\operatorname{det}\left(p_{i}, q_{i+1}\right)+\operatorname{det}\left(q_{i}, p_{i+1}\right)\right) \text {. }
$$

This formula describes the symmetric bilinear form induced by the area functional in any vector space of polygons with $N$ vertices.

If either $P$ or $Q$ has zero edges (i.e., multiple vertices), the vertices of $\partial(\lambda K+\mu L)$ need not equal $\lambda p_{i}+\mu q_{i}$, but if they do, (3) is valid.

Note that arbitrary polygons $\partial K, \partial L$ can be seen as parallel polygons, as illustrated by Fig. 1: We label the vertices $r_{0}, \ldots, r_{N-1}$ of $\partial(K+L)$ consecutively and subsequently give (possibly multiple) indices $p_{0}, \ldots, p_{N-1}$ and $q_{0}, \ldots, q_{N-1}$ to the vertices of $\partial K$ and $\partial L$ such that $p_{i}+q_{i}=r_{i}$. Then all three boundaries of $K, L$, $K+L$, and in fact $\lambda K+\mu L$ for $\lambda, \mu \geq 0$ are described by parallel polygons, and (3) can be used for computing the mixed area in the sense of (1).

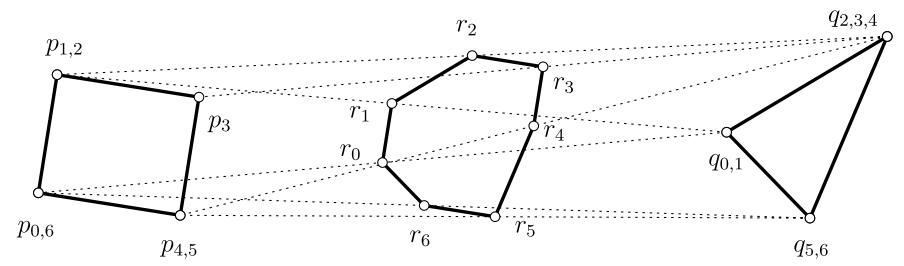

Fig. 1 Labeling vertices of $\partial K$ and $\partial L$ with aid of $\partial(K+L)$ such that they become parallel 


\subsection{The Oriented Mixed Area}

The polygons parallel to a given polygon $P=\left(p_{0}, \ldots, p_{N-1}\right)$, not necessarily convex, constitute a vector space under vertex-wise addition and scalar multiplication. Its dimension equals $N+\#\left\{i \mid p_{i}=p_{i+1}\right\}$. If there are no zero edges, we use the symbol

$$
\mathcal{P}(P)=\left\{\left(q_{0}, \ldots, q_{N-1}\right) \mid q_{i+1}-q_{i}=\lambda_{i}\left(p_{i+1}-p_{i}\right), 0 \leq i<N\right\} / \mathbb{R}^{2}
$$

for the vector space of polygons parallel to $P$, modulo parallel translations. Then $\operatorname{dim} \mathcal{P}(P)=N-2$. If both $P$ and $Q$ have zero edges, still $P+Q$ might not have, and consequently $P, Q, P+Q \in \mathcal{P}(P+Q)$. The expression defined by formula (3) is translation invariant. Following [6], we define:

Definition 1 For parallel polygons $P=\left(p_{0}, \ldots, p_{N-1}\right)$ and $Q=\left(q_{0}, \ldots, q_{N-1}\right)$, the oriented mixed area is given by the bilinear form (3).

Apparently the oriented mixed area, which extends the concept of mixed area for convex domains, is the bilinear form associated with the quadratic form measuring oriented area by Leibniz' sector formula.

\subsection{Discrete Minimal Surfaces}

The curvature theory presented in [6] deals with parallel meshes, which means a pair $(\Sigma, \Phi)$ of polyhedral surfaces having the same combinatorics and such that corresponding edges are parallel. $\Sigma$ is viewed as the Gauss image of $\Phi$. The definition of parallelity of polygons extends to polygons which lie in parallel planes; in order to employ (3) for the computation of mixed area they have to be moved to a common plane by parallel translation. If $P$ is a face and $Q$ the corresponding face in the Gauss image, then $P$ is assigned the mean and Gaussian curvatures

$$
H_{P}=-\frac{\operatorname{area}(P, Q)}{\operatorname{area}(P)}, \quad K_{P}=\frac{\operatorname{area}(Q)}{\operatorname{area}(P)} .
$$

For this reason, vanishing mixed area of parallel polygons is characteristic for discrete minimal surfaces. The following result of [6], illustrated in Fig. 2, is basic for the construction of minimal surfaces with regular quad mesh combinatorics:

Proposition 2 Parallel quadrilaterals $P=\left(p_{0}, p_{1}, p_{2}, p_{3}\right)$ and $Q=\left(q_{0}, q_{1}, q_{2}, q_{3}\right)$ have vanishing mixed area if and only if the diagonals $p_{0} p_{2}$ and $q_{1} q_{3}$ are parallel, which is equivalent to the diagonals $p_{1} p_{3}$ and $q_{0} q_{2}$ being parallel.

Fig. 2 For nondegenerate parallel quads $p_{0}, \ldots, p_{3}$ and $q_{0}, \ldots, q_{3}$, parallel of one nonzero diagonal characterizes vanishing mixed area $\left(q_{0} q_{2} \| q_{1} q_{3}\right.$, or $\left(q_{1} q_{3} \| q_{0} q_{2}\right)$
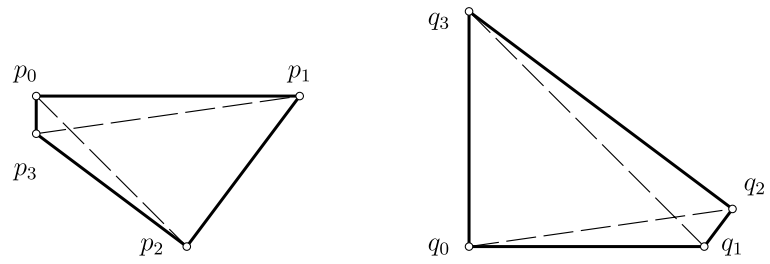
This condition actually discretizes the Christoffel duality between a minimal surface and its spherical Gauss image [6]. We therefore call a polyhedral surface and its parallel Gauss image where all mixed areas vanish, a Christoffel dual pair. It is an interesting fact that Proposition 2 applies to several constructions of discrete minimal surfaces based on quadrilaterals, namely the ones of $[1,4]$. The present paper, which deals with vanishing mixed area in general, is focused on hexagonal meshes.

\section{Properties of the Oriented Mixed Area}

The main result of the present paper is the recursion formula of Theorem 6 below, which for parallel polygons $P, Q$ with an even number of vertices shows that $\operatorname{area}(P, Q)=\operatorname{area}\left(P^{*}, Q^{*}\right)$, where $P^{*}, Q^{*}$ are derived polygons which have only the half number of vertices. It is used in Sect. 3 to derive geometric characterizations of parallel polygons with vanishing mixed area.

\subsection{Formulas for the Mixed Area}

There are equivalent formulae for the oriented mixed area of parallel polygons which take advantage of parallelity:

Lemma 3 The bilinear form of (3) is alternatively expressed as

$$
\sum_{i \in\{0,1, \ldots, N-1\}}\left(\operatorname{det}\left(p_{i}, q_{i+1}\right)+\operatorname{det}\left(q_{i}, p_{i+1}\right)\right)=\sum_{i \in\{0,1, \ldots, N-1\}} \operatorname{det}\left(p_{i}, q_{i+1}-q_{i-1}\right)
$$

(indices modulo $N$ ). For $P, Q$ parallel and an even number of vertices,

$$
\begin{aligned}
2 \operatorname{area}(P, Q) & =\sum_{i \in\{0,2, \ldots, N-2\}} \operatorname{det}\left(p_{i}, q_{i+1}-q_{i-1}\right) \\
& =\sum_{i \in\{1,3, \ldots, N-1\}} \operatorname{det}\left(p_{i}, q_{i+1}-q_{i-1}\right) .
\end{aligned}
$$

Proof The first equality is found by rearranging indices:

$$
\sum_{i} \operatorname{det}\left(p_{i}, q_{i+1}\right)+\sum_{i} \operatorname{det}\left(q_{i}, p_{i+1}\right)=\sum_{i} \operatorname{det}\left(p_{i}, q_{i+1}\right)+\sum_{i} \operatorname{det}\left(q_{i-1}, p_{i}\right) .
$$

In order to show the second equality, we observe that parallelity of $P$ and $Q$ implies $\operatorname{det}\left(p_{i+1}-p_{i}, q_{i+1}-q_{i}\right)=0$. Therefore,

$$
\begin{aligned}
& \sum_{i \text { even }} \operatorname{det}\left(p_{i}, q_{i+1}-q_{i-1}\right) \\
& \quad=\sum_{i \text { even }}\left(\operatorname{det}\left(p_{i}, q_{i+1}-q_{i}\right)+\operatorname{det}\left(p_{i}, q_{i}-q_{i-1}\right)\right)
\end{aligned}
$$




$$
\begin{aligned}
& =\sum_{i \text { even }}\left(\operatorname{det}\left(p_{i}+\left(p_{i+1}-p_{i}\right), q_{i+1}-q_{i}\right)+\operatorname{det}\left(p_{i}+\left(p_{i-1}-p_{i}\right), q_{i}-q_{i-1}\right)\right) \\
& =\sum_{i \text { even }}\left(\operatorname{det}\left(p_{i+1}, q_{i+1}-q_{i}\right)+\operatorname{det}\left(p_{i-1}, q_{i}-q_{i-1}\right)\right) \\
& =\sum_{i \text { even }}\left(\operatorname{det}\left(p_{i+1}, q_{i+1}-q_{i}\right)+\operatorname{det}\left(p_{i+1}, q_{i+2}-q_{i+1}\right)\right) \\
& =\sum_{i \text { even }} \operatorname{det}\left(p_{i+1}, q_{i+2}-q_{i}\right)=\sum_{i \text { odd }} \operatorname{det}\left(p_{i}, q_{i+1}-q_{i-1}\right) .
\end{aligned}
$$

It follows that the expression for $4 \operatorname{area}(P, Q)$ according to (3) reads

$$
\left(\sum_{i \text { even }}+\sum_{i \text { odd }}\right) \operatorname{det}\left(p_{i}, q_{i+1}-q_{i-1}\right)=\sum_{i \text { even }} 2 \operatorname{det}\left(p_{i}, q_{i+1}-q_{i-1}\right),
$$

which is what we wanted to prove.

We have now obtained an expression for the mixed area which makes use only of the vertices $p_{0}, p_{2}, \ldots$ and $q_{1}, q_{3}, \ldots$, which however does not have a direct relation to the areas associated with polygons $\left(p_{0}, p_{2}, \ldots\right)$ and $\left(q_{1}, q_{3}, \ldots\right)$.

\subsection{Construction of a Derived Polygon}

For the following construction of derived polygons, the polygons $P, Q$ must have an even number $N$ of vertices, and certain diagonals of $P$ are forbidden to be parallel. We used the notation $[v]$ for the one-dimensional subspace spanned by the vector $v$.

Definition 4 Assume that a pair of polygons $P=\left(p_{0}, \ldots, p_{N-1}\right)$ and $Q=\left(q_{0}, \ldots\right.$, $\left.q_{N-1}\right)$, where $N$ is even, is such that

$$
p_{i}-p_{i-2} \text { and } p_{i+2}-p_{i} \text { are linearly independent } \text { for } i=0,2,4, \ldots
$$

(indices are taken modulo $N$ ). Then a pair of derived polygons $P^{*}, Q^{*}$ is constructed such that $P^{*}$ consists of every other vertex of $P$, while the vertices of $Q^{*}$ are found by parallel translating diagonals of $P$ through points of $Q$ :

$$
p_{i}^{*}=p_{2 i}, \quad q_{i}^{*}=\left(q_{2 i+1}+\left[p_{2 i+2}-p_{2 i}\right]\right) \cap\left(q_{2 i-1}+\left[p_{2 i}-p_{2 i-2}\right]\right) .
$$

An index shift of 1 yields an analogous derived pair $\left(P^{* *}, Q^{* *}\right)$ based on the odd vertices of $P: p_{i}^{* *}=p_{2 i+1}$ and $q_{i}^{* *}=\left(q_{2 i+2}+\left[p_{2 i+3}-p_{2 i+1}\right]\right) \cap\left(q_{2 i}+\left[p_{2 i+1}-\right.\right.$ $\left.p_{2 i-1}\right]$ ), provided that the lines employed in this intersection are not parallel.

This construction is illustrated by Figs. 3 and 4 . Note that if $Q^{*}$ cannot be constructed because (7) is not fulfilled, still $Q^{* *}$ may be constructible. For quadrilaterals, (7) is never fulfilled, even after an index shift. We will be able to treat quadrilaterals as degenerate hexagons. 

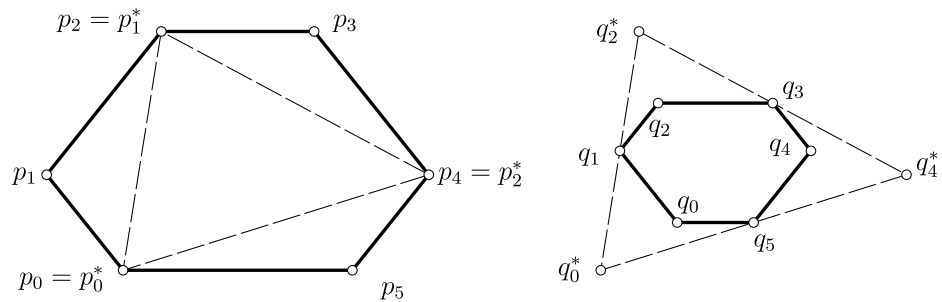

Fig. 3 A pair of polygons $P, Q$ and the derived polygons $P^{*}, Q^{*}$
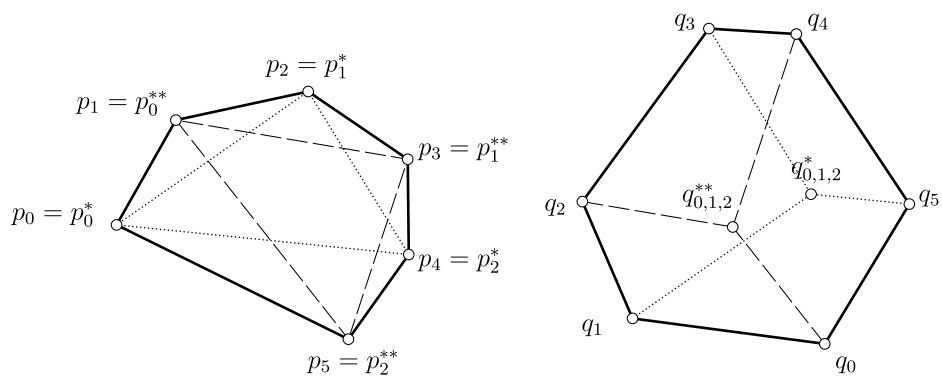

Fig. 4 Illustration of Lemma 5. Polygons $P^{*}, Q^{*}$ are orthogonal with respect to the bilinear form defined by the oriented are if both derived polygons $Q^{*}$ and $Q^{* *}$ degenerate into a point

\subsection{Nonparallel Polygons}

The bilinear function of (3) which measures the oriented mixed area for parallel polygons can be evaluated for arbitrary pairs of polygons, without having a geometric meaning as mixed area in the classical sense. It still extends the quadratic functional which measures the oriented area by Leibniz' sector formula. The definition of derived polygons according to Definition 4 is not restricted to parallel polygons.

We here record a nice geometric property of this functional:

Lemma 5 Polygons $P, Q$ are orthogonal with respect to the bilinear function (3) if both derived polygons $Q^{*}$ and $Q^{* *}$ with respect to even and odd indices degenerate into points $q^{*}$ and $q^{* *}$, respectively.

Proof By Lemma 3, we have to show that $\sum_{0 \leq i<N} \operatorname{det}\left(p_{i}, q_{i+1}-q_{i-1}\right)=0$. Our assumption on the degeneracy of derived polygons means that there are real numbers $\lambda_{i}$ with

$$
\begin{aligned}
& q^{*}=q_{i}+\lambda_{i}\left(p_{i-1}-p_{i+1}\right) \quad \text { for } i \text { even, } \\
& q^{* *}=q_{i}+\lambda_{i}\left(p_{i-1}-p_{i+1}\right) \quad \text { for } i \text { odd. } \\
& \quad \Longrightarrow \quad q_{i+1}-q_{i-1}=\lambda_{i-1}\left(p_{i-2}-p_{i}\right)-\lambda_{i+1}\left(p_{i}-p_{i+2}\right) \quad \text { for all } i .
\end{aligned}
$$


We use this equality to evaluate (3):

$$
\begin{aligned}
& \sum_{0 \leq i<N} \operatorname{det}\left(p_{i}, q_{i+1}-q_{i-1}\right) \\
& \quad=\sum_{0 \leq i<N} \operatorname{det}\left(p_{i}, \lambda_{i-1}\left(p_{i-2}-p_{i}\right)-\lambda_{i+1}\left(p_{i}-p_{i+2}\right)\right) \\
& \quad=\sum_{0 \leq i<N} \lambda_{i} \operatorname{det}\left(p_{i+1}, p_{i-1}-p_{i+1}\right)-\sum_{0 \leq i<N} \lambda_{i} \operatorname{det}\left(p_{i-1}, p_{i-1}-p_{i+1}\right) \\
& \quad=\sum_{0 \leq i<N} \lambda_{i} \operatorname{det}\left(p_{i+1}-p_{i-1}, p_{i-1}-p_{i+1}\right)=0
\end{aligned}
$$

(indices are taken modulo $N$ ). This concludes the proof.

\subsection{Mixed Area of Derived Polygons}

The following theorem is the main technical contribution of the present paper. It is the basis of geometric characterizations of polygons pairs with vanishing mixed area, and therefore the basis of constructions of discrete minimal surfaces.

Theorem 6 For any pair of parallel polygons $P, Q$ with an even number of vertices, the oriented mixed area is unaffected by the passage to derived polygons: $\operatorname{area}(P, Q)=\operatorname{area}\left(P^{*}, Q^{*}\right)=\operatorname{area}\left(P^{* *}, Q^{* *}\right)$ whenever the conditions of Definition 4 , which allow construction of $Q^{*}$ or $Q^{* *}$, are fulfilled.

Proof It is obviously sufficient to show the result for $P^{*}$ and $Q^{*}$. An elementary computation yields the coordinates of $Q^{*}$ 's vertices $q_{i}^{*}$ :

$$
\begin{aligned}
& q_{i}^{*}=\frac{1}{\gamma_{i}}\left(\alpha_{i}\left(p_{i+1}^{*}-p_{i}^{*}\right)+\beta_{i}\left(p_{i}^{*}-p_{i-1}^{*}\right)\right), \quad \text { where } \\
& \alpha_{i}=\operatorname{det}\left(q_{2 i-1}, p_{i}^{*}-p_{i-1}^{*}\right), \quad \beta_{i}=\operatorname{det}\left(p_{i+1}^{*}-p_{i}^{*}, q_{2 i+1}\right), \\
& \gamma_{i}=\operatorname{det}\left(p_{i+1}^{*}-p_{i}^{*}, p_{i}^{*}-p_{i-1}^{*}\right) .
\end{aligned}
$$

We introduce the notation $\Delta_{j}^{k}=\operatorname{det}\left(p_{j}^{*}, p_{k}^{*}\right)$, which has the properties

$$
\Delta_{j}^{j}=0, \quad \Delta_{i}^{j}=-\Delta_{j}^{i}, \quad \gamma_{i}=\Delta_{i+1}^{i}-\Delta_{i+1}^{i-1}+\Delta_{i}^{i-1} .
$$

For the mixed area of $P^{*}$ and $Q^{*}$, we consider the following sum, where indices $i$ range from 0 to $N / 2-1$, and indices $j$ range from 0 to $N-1$ :

$$
\begin{aligned}
& \sum_{i} \operatorname{det}\left(q_{i}^{*}, p_{i+1}^{*}-p_{i-1}^{*}\right) \\
& \quad=\sum_{i} \frac{1}{\gamma_{i}} \operatorname{det}\left(\alpha_{i}\left(p_{i+1}^{*}-p_{i}^{*}\right)+\beta_{i}\left(p_{i}^{*}-p_{i-1}^{*}\right), p_{i+1}^{*}-p_{i-1}^{*}\right)
\end{aligned}
$$




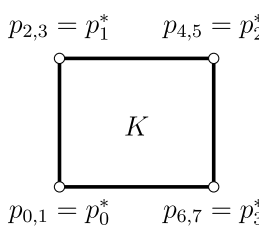

(a)

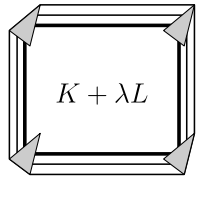

(b) (c)

(d)

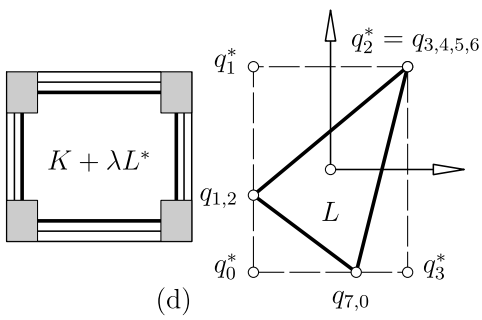

Fig. 5 An explanation of the fact $\operatorname{area}(P, Q)=\operatorname{area}\left(P^{*}, Q^{*}\right)$ for artificially parallel polygons which occur as boundaries $P=\partial K$ and $Q=\partial L$. (a) Both polygons $P$ and $P^{*}$ describe the boundary $\partial K$. (b) Convex domains $K+\lambda L$, where $\lambda$ equals 0.1 and 0.2 . (c) The same for $L^{*}$ instead of $L$, where the boundary $\partial L^{*}$ is given by the polygon $Q^{*}$. (d) Polygons $Q$ and $Q^{*}$. Obviously the linearly growing part of area $(K+\lambda L)$ along the edges of $\partial K$ is not affected if we replace $L$ by $L^{*}$

$$
\begin{aligned}
& =\sum_{i} \frac{1}{\gamma_{i}}\left(\alpha_{i}\left(-\Delta_{i+1}^{i-1}-\Delta_{i}^{i+1}+\Delta_{i}^{i-1}\right)+\beta_{i}\left(\Delta_{i}^{i+1}-\Delta_{i}^{i-1}-\Delta_{i-1}^{i+1}\right)\right) \\
& =\sum_{i} \frac{1}{\gamma_{i}}\left(\alpha_{i} \gamma_{i}-\beta_{i} \gamma_{i}\right)=\sum_{i}\left(\operatorname{det}\left(q_{2 i-1}, p_{i}^{*}-p_{i-1}^{*}\right)-\operatorname{det}\left(p_{i+1}^{*}-p_{i}^{*}, q_{2 i+1}\right)\right) \\
& =\sum_{j \text { even }}\left(\operatorname{det}\left(q_{j-1}, p_{j}-p_{j-2}\right)+\operatorname{det}\left(q_{j+1}, p_{j+2}-p_{j}\right)\right) .
\end{aligned}
$$

An index shift shows that $\sum_{j \text { even }} \operatorname{det}\left(q_{j-1}, p_{j}-p_{j-2}\right)=\sum_{j \text { even }} \operatorname{det}\left(q_{j+1}\right.$, $\left.p_{j+2}-p_{j}\right)=\sum_{j \text { odd }} \operatorname{det}\left(q_{j}, p_{j+1}-p_{j-1}\right)$, so we conclude that

$$
\operatorname{area}\left(P^{*}, Q^{*}\right)=\frac{1}{4} \sum_{i} \operatorname{det}\left(q_{i}^{*}, p_{i+1}^{*}-p_{i-1}^{*}\right)=\frac{1}{2} \sum_{j \text { odd }} \operatorname{det}\left(q_{j}, p_{j+1}-p_{j-1}\right)
$$

By Lemma 3, this expression equals area $(P, Q)$, which concludes the proof.

This result concerning the mixed area of parallel polygons is strong in reducing the number of vertices to deal with by a factor 2 . However, if we apply it to two arbitrary polygons, which are made artificially parallel like those of Fig. 1, we only obtain a fact which is obvious from the definition of mixed area anyway. As illustrated by Fig. 5, $Q^{*}$ then describes the boundary of the domain $L^{*}$ constructed from $K$ and $L$ as the intersection of all supporting half-spaces of $L$ whose boundary is parallel to an edge of $K$. Obviously, area $(K, L)=\operatorname{area}\left(K, L^{*}\right)$.

\section{Vanishing Mixed Areas}

This section applies Theorem 6 to parallel polygons $P, Q$ whose mixed area vanishes. As mentioned in Sect. 1.3, such pairs of polygons are especially interesting for the construction of discrete minimal surfaces. 
Fig. 6 A Pappos hexagon does not fulfill the requirements of Definition 4, and so Theorem 7 is not applicable to this pair of parallel Pappos hexagons with $\operatorname{area}(P, Q)=0$ (we must apply Proposition 8 instead)

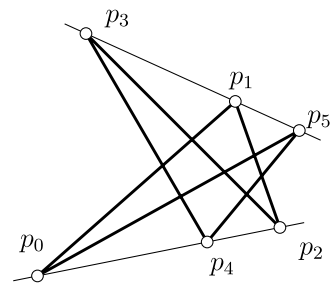

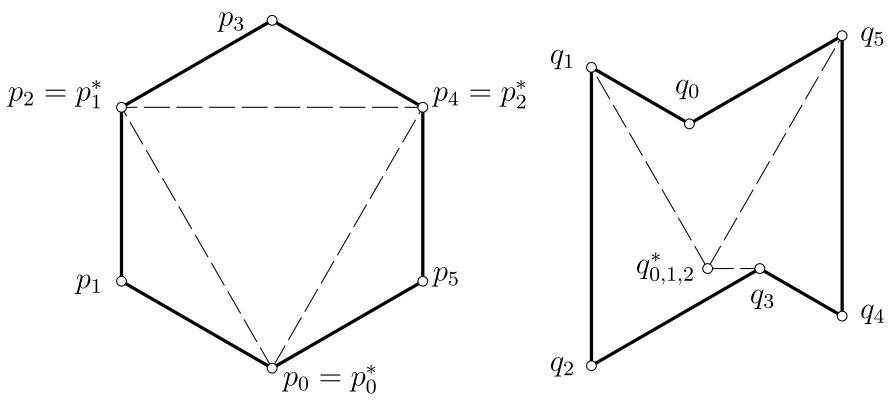

Fig. 7 Parallel hexagons $P, Q$ with vanishing mixed area (the derived triangle $Q^{*}$ degenerates)

\subsection{Vanishing Mixed Area for Parallel Hexagons}

Here we treat both quadrilaterals and 5-gons as special hexagons. Before we derive a geometric criterion for the vanishing of mixed area for parallel hexagons (Fig. 7), we discuss pairs $P, Q$ of hexagons where Theorem 6 cannot be applied, because neither derived polygon $Q^{*}, Q^{* *}$ can be constructed. Obviously, this is the case if and only both $p_{0}, p_{2}, p_{4}$ and $p_{1}, p_{3}, p_{5}$ are collinear, i.e., $P$ is a Pappos hexagon (see Fig. 6). Exchanging the role of $P$ and $Q$ helps, except for the case that both $q_{0}, q_{2}, q_{4}$ and $q_{1}, q_{3}, q_{5}$ are collinear.

Theorem 7 For parallel hexagons $P, Q$ where one, say $P$, is not a Pappos hexagon, there is a labeling of vertices such that the derived polygons $P^{*}, Q^{*}$ can be constructed. Then area $(P, Q)=0$ if and only if all vertices of the triangle $Q^{*}$ coincide, i.e., if the lines

$$
q_{1}+\left[p_{2}-p_{0}\right], \quad q_{3}+\left[p_{4}-p_{2}\right], \quad q_{5}+\left[p_{0}-p_{4}\right]
$$

intersect in a common point.

Proof The derived polygons $P^{*}, Q^{*}$ are parallel triangles with $Q^{*}=\lambda P^{*}+a$. It follows that $\operatorname{area}\left(P^{*}, Q^{*}\right)=\lambda$ area $\left(P^{*}\right)$. By non-collinearity of $P^{*}$, this area vanishes if and only if $\lambda=0$.

The case of two Pappos hexagons has to be treated separately: 


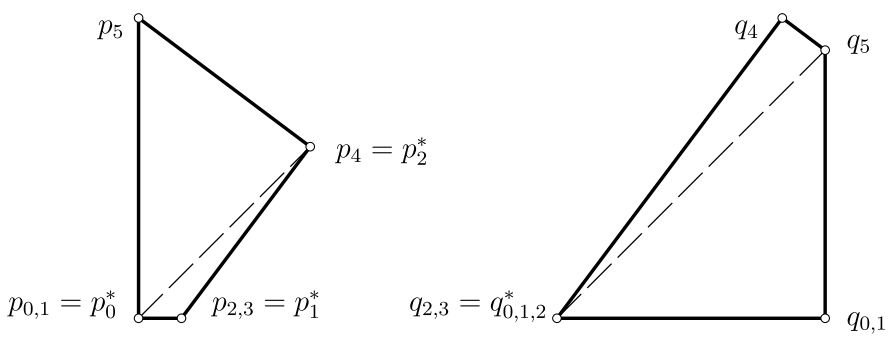

Fig. 8 Quadrilaterals with antiparallel diagonals are degenerate hexagons $P, Q$, where the triangle $Q^{*}$ degenerates into a point

Proposition 8 Suppose that $P, Q$ are two parallel hexagons such that $p_{1}, p_{3}, p_{5}$ as well as $q_{0}, q_{2}, q_{4}$ are collinear (this includes the case of parallel Pappos hexagons). Then $\operatorname{area}(P, Q)=0$ in exactly the following cases:

(i) $p_{1}=p_{3}=p_{5}$ or $q_{0}=q_{2}=q_{4}$.

(ii) The triples $\left(p_{1}, p_{3}, p_{5}\right)$ and $\left(q_{4}, q_{0}, q_{2}\right)$ are affinely equivalent.

(iii) The lines which carry $p_{1}, p_{3}, p_{5}$ and $q_{0}, q_{2}, q_{4}$ are parallel.

Proof By parallel translation we can achieve that the straight lines pass through the origin of the coordinate system, so there are $v, w \in \mathbb{R}^{2} \backslash 0$ with $p_{i}=\lambda_{i} v$ for $i=1,3,5$ and $q_{j}=\mu_{j} w$ for $j=0,2,4$. By (5),

$$
\begin{aligned}
2 \operatorname{area}(P, Q) & =\sum_{i=1,3,5} \operatorname{det}\left(\lambda_{i} v,\left(\mu_{i+1}-\mu_{i-1}\right) w\right) \\
& =\operatorname{det}(v, w)\left(\lambda_{1}\left(\mu_{2}-\mu_{0}\right)+\lambda_{3}\left(\mu_{4}-\mu_{2}\right)+\lambda_{5}\left(\mu_{0}-\mu_{4}\right)\right) \\
& =\operatorname{det}(v, w)\left(\left(\lambda_{1}-\lambda_{3}\right)\left(\mu_{2}-\mu_{4}\right)-\left(\lambda_{1}-\lambda_{5}\right)\left(\mu_{0}-\mu_{4}\right)\right) .
\end{aligned}
$$

In case (iii) we have $\operatorname{det}(v, w)=0$, so $\operatorname{area}(P, Q)=0$. In case (i) we have $\lambda_{1}=$ $\lambda_{3}=\lambda_{5}$ or $\mu_{0}=\mu_{2}=\mu_{4}$, which likewise implies vanishing mixed area. We now assume that we have neither case (i) nor case (iii). Then

$$
\operatorname{area}(P, Q)=0 \quad \Longleftrightarrow \quad\left(\lambda_{1}-\lambda_{3}\right):\left(\lambda_{1}-\lambda_{5}\right)=\left(\mu_{4}-\mu_{0}\right):\left(\mu_{4}-\mu_{2}\right),
$$

i.e., if and only if the ratio of collinear points $p_{1}, p_{3}, p_{5}$ equals the ratio of collinear points $q_{4}, q_{0}, q_{2}$. This is equivalent to (ii).

We refrain from an exhaustive discussion of cases. An example of two parallel Pappos hexagons with vanishing mixed area is shown by Fig. 6 .

\subsection{Vanishing Mixed Area for 4- and 5-gons as Degenerate 6-gons}

Two parallel quadrilaterals are converted to parallel hexagons if we count two pairs of corresponding vertices twice. This operation does not change the mixed area, and Theorem 7 immediately gives the known result of Proposition 2 that vanishing mixed area is characterized by anti-parallelity of diagonals (see Figs. 2 and 8). Anyway 

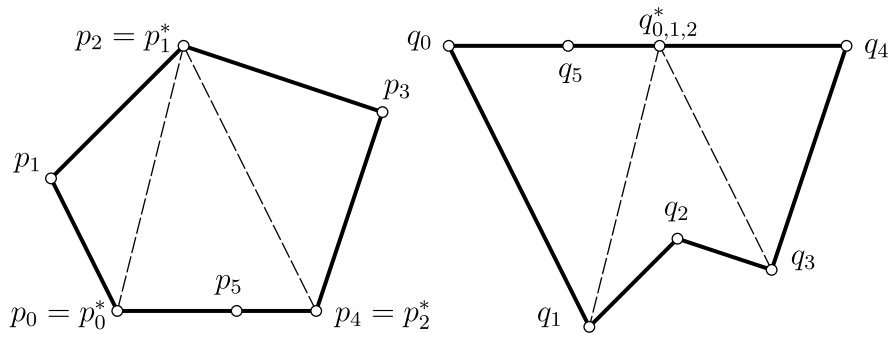

Fig. 9 A pair of parallel 5-gons $P, Q$ seen as hexagons and a geometric characterization of vanishing mixed area which follows from this interpretation. Index shifts produce equivalent configurations
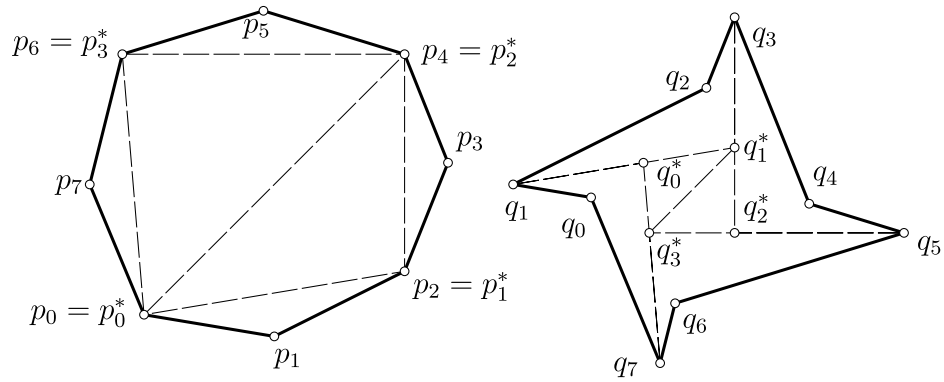

Fig. 10 Parallel 8-gons $P, Q$ with vanishing mixed area. The derived polygons $P^{*}, Q^{*}$ are parallel quads with area $\left(P^{*}, Q^{*}\right)=0$ and consequently antiparallel diagonals

Proposition 2 follows directly from Lemma 3, because for parallel quads, (6) expands to $\operatorname{area}(P, Q)=\operatorname{det}\left(p_{0}-p_{2}, q_{1}-q_{3}\right)$. For 5-gons, we have the following result:

Corollary 9 The oriented mixed area of two parallel 5-gons $P=\left(p_{0}, \ldots, p_{4}\right)$ and $Q=\left(q_{0}, \ldots, q_{4}\right)$ vanishes if both of them are contained in a straight line. If this is not the case, area $(P, Q)=0$ is characterized by the following geometric condition for one index $i$ (and equivalently for all indices $i$ ): The lines

$$
q_{i+1}+\left[p_{i+2}-p_{i}\right] \text { and } q_{i+3}+\left[p_{i+4}-p_{i+2}\right]
$$

meet on the edge $q_{i+4} q_{i}$, where indices are taken modulo 5 .

Proof It is sufficient to assume that $p_{0}, p_{1}, p_{4}$ are not collinear and show the result for the case $i=0$ illustrated by Fig. 9. By introducing vertices $p_{5}, q_{5}$ in the edges $p_{4} p_{0}$ and $q_{4} q_{0}$, resp., we convert both $P$ and $Q$ into parallel hexagons, and Theorem 7 is applicable. The statement that $Q^{*}$ degenerates to a point translates to the statement we want to show.

\subsection{Vanishing Mixed Area for 8-, 7-, and 6-gons (Again)}

Theorem 6 is recursively applicable, but the number of cases to be distinguished because diagonals are parallel and derived polygons cannot be constructed becomes 


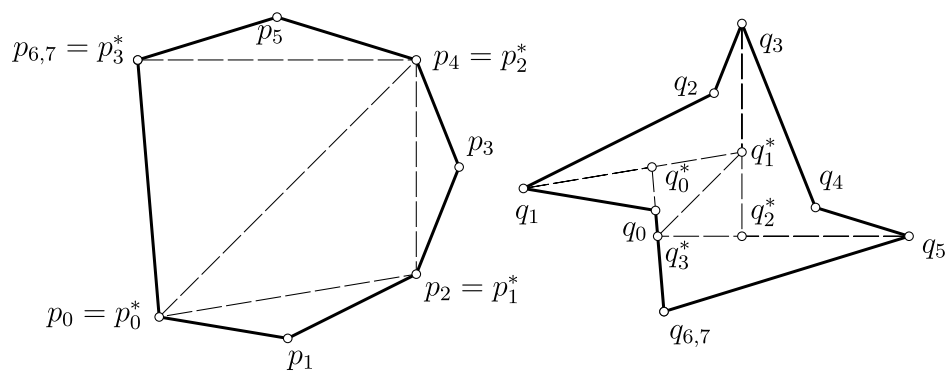

Fig. 11 Parallel 7-gons $P, Q$ with vanishing mixed area, which are interpreted as 8-gons with $p_{6}=p_{7}$ and $q_{6}=q_{7}$, respectively. The derived polygons $P^{*}, Q^{*}$ are parallel quads with area $\left(P^{*}, Q^{*}\right)=0$ and consequently antiparallel diagonals

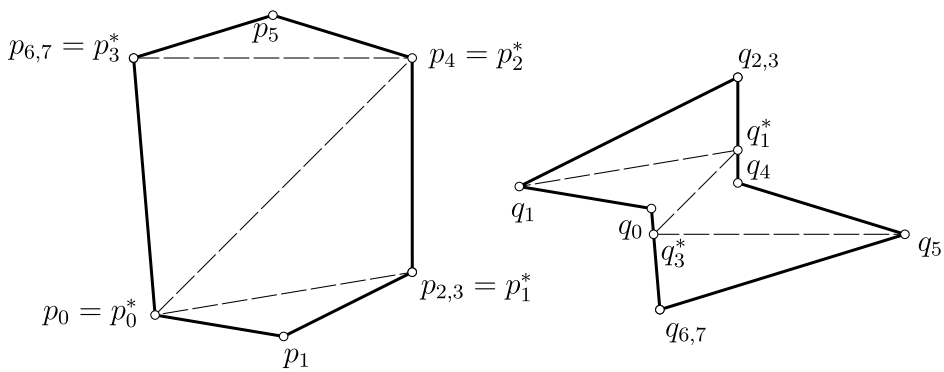

Fig. 12 Parallel hexagons $P, Q$ with vanishing mixed area, which are interpreted as 8-gons with $p_{6}=p_{7}, p_{2}=p_{3}$ and $q_{6}=q_{7}, q_{2}=q_{3}$, respectively. The derived polygons $P^{*}, Q^{*}$ are parallel quads with area $\left(P^{*}, Q^{*}\right)=0$ and consequently antiparallel diagonals. For a geometric characterization, only one half of $Q^{*}$ is needed

greater. The following result for generic 8-gons follows directly from Theorem 6 and Proposition 2 (see Fig. 10).

Corollary 10 Parallel 8-gons $P, Q$ where the derived polygons $P^{*}, Q^{*}$ can be constructed have vanishing mixed area exactly in the following three cases: $(i)$ both sets $\left\{p_{0}, p_{2}, p_{4}, p_{6}\right\}$ and $\left\{q_{0}^{*}, q_{1}^{*}, q_{2}^{*}, q_{3}^{*}\right\}$ are contained in a straight line; (ii) $p_{0} \neq p_{4}$, and $p_{0} p_{4}$ is parallel to $q_{1}^{*} p_{3}^{*}$; (iii) $p_{2} \neq p_{6}$, and $p_{2} p_{6}$ is parallel to $q_{0}^{*} p_{2}^{*}$.

The obvious way to treat parallel 7-gons is as 8-gons with two coincident vertices: We simply add an 8th vertex $p_{7}=p_{6}$ and $q_{7}=q_{6}$ to each an apply Corollary 10 . This is illustrated by Fig. 11. It is interesting to observe that also for hexagons we get again an easy geometric condition if we treat them as 8-gons with two coincident vertices (see Fig. 12). Disregarding special cases, we have:

Corollary 11 The parallel hexagons $\left(p_{0}, p_{1}, p_{2}, p_{4}, p_{5}, p_{6}\right)$ and $\left(q_{0}, q_{1}, q_{2}, q_{4}\right.$, $\left.q_{5}, q_{6}\right)$ generically have zero oriented mixed area if and only if $p_{0} \vee p_{4} \| q_{1}^{*} \vee q_{3}^{*}$, where

$$
q_{1}^{*}=\left(q_{1}+\left[p_{2}-p_{0}\right]\right) \cap\left(q_{2} \vee q_{4}\right), \quad q_{3}^{*}=\left(q_{5}+\left[p_{4}-p_{6}\right]\right) \cap\left(q_{6} \vee q_{0}\right) .
$$


Proof The polygons are extended to 8-gons $P, Q$ with the same mixed area by letting $p_{3}:=p_{2}, p_{7}:=p_{6}, q_{3}:=q_{2}$, and $q_{7}:=q_{6}$. The derived quadrilateral $Q^{*}$ according to Corollary 10 has the two vertices $q_{1}^{*}, q_{3}^{*}$, so the result follows.

\section{Discrete Minimal Surfaces}

Section 1.3 already introduced in general terms the definitions of curvature according to [6]. Recall that a polyhedral surface $\Phi$ is a discrete minimal surface with respect to a Gauss image $\Sigma$ if $\Phi, \Sigma$ are parallel meshes such that corresponding faces have vanishing mixed area. We view $\Phi$ as a discrete Christoffel dual of $\Sigma$, and vice versa.

Not every quadrilateral mesh $\Sigma$ with planar faces (i.e., a polyhedral surface with regular grid combinatorics) has a Christoffel dual $\Phi$ : It turns out that exactly the Koenigs nets (see [2,3]) have this property.

\subsection{Construction of Hexagonal Minimal Surfaces}

Figures 13, 14 illustrate a parallel pair $(\Sigma, \Phi)$ of meshes (for more details, see the example below). The faces of $\Sigma$ are tangent to the unit sphere, so it makes sense to consider $\Sigma$ as a discrete Gauss image of $\Phi$. As $\Phi$ has been constructed such that the mixed area of corresponding faces vanishes, $\Phi$ is discrete minimal. It should be mentioned in this place that the exact relation between the discrete Gauss image $\Sigma$ and the unit sphere $S^{2}$ can be of a different nature: We could also require that vertices of $\Sigma$ lie in $S^{2}$, or its edges are tangent to $S^{2}$. The three cases of vertices, edges, and faces having an exact tangency relation with $S^{2}$ correspond to the mesh $\Phi$ having offset meshes $\Phi+d \Sigma$ at constant vertex-vertex distance $d$, or edge-edge distance $d$, or face-face distance $d[6,7]$. Note that the property of vanishing mixed area does not only occur between minimal surface and Gauss image but also between a surface $\Phi$ of constant mean curvature $H$ with respect to a Gauss image $\Sigma$ and its offset $\Phi+\frac{1}{H} \Sigma$.
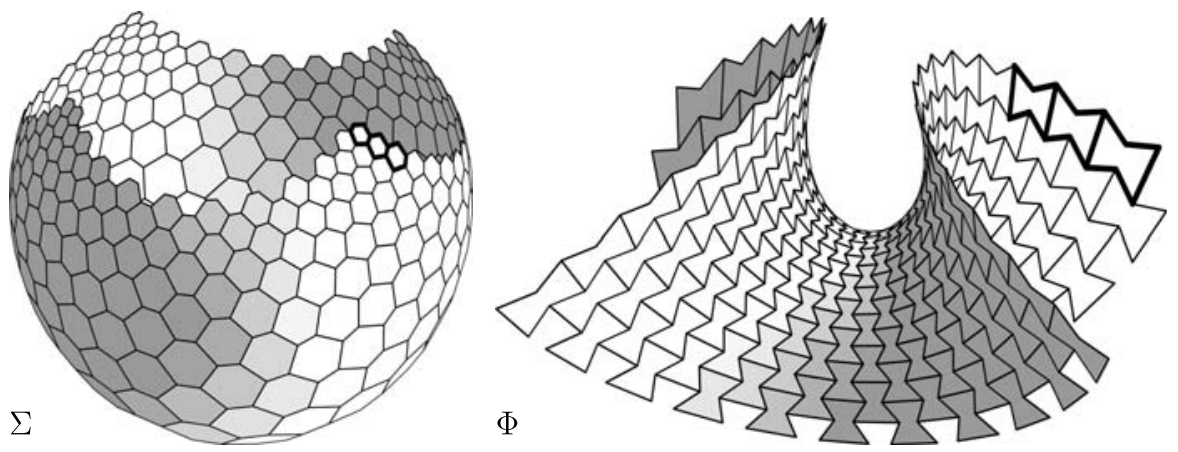

Fig. 13 A discrete Enneper's surface $\Phi$ in the shape of a hexagonal mesh, which corresponds to a spherical mesh $\Sigma$ by a discrete Christoffel duality. The marked hexagons correspond to each other 

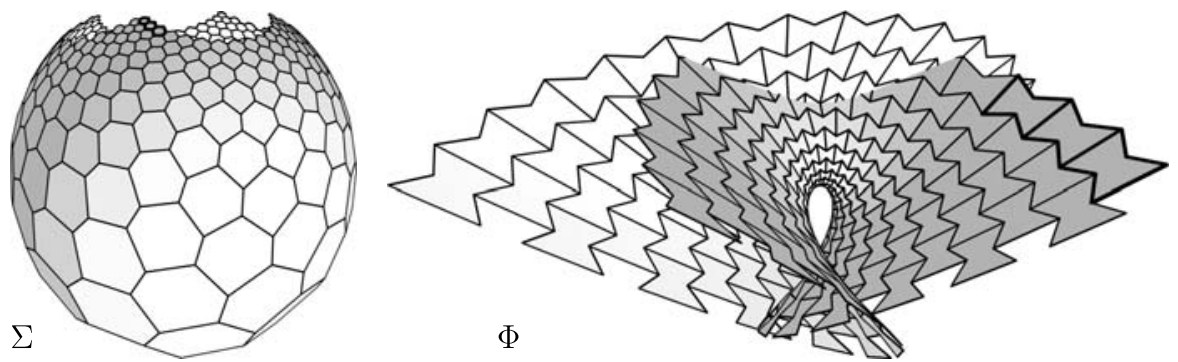

Fig. 14 A discrete Enneper's surface created in the same way as Fig. 13 but with a different size of the spherical mesh $\Sigma$. Here $\Phi$ has self-intersections. The viewpoint is different from that of Fig. 13

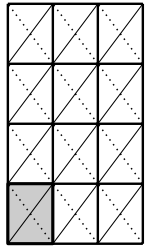

(a)

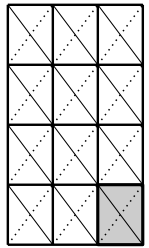

(b)

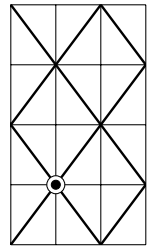

(c)

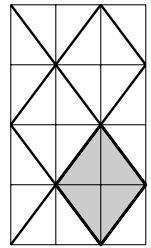

(d)

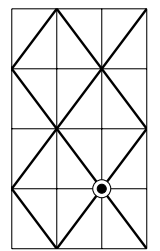

(e)

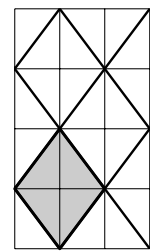

(f)

Fig. 15 Reciprocal-parallel meshes derived from a quadrilateral mesh $\Sigma$ and its Christoffel dual mesh $\Phi$, which is combinatorially equivalent such that corresponding quadrilaterals $P$ and $Q$ have vanishing mixed area. From left: (a) Mesh $\Sigma$ with face $P$. (b) Mesh $\Phi$ with face $Q$ corresponding to $P$. (c) Quadrilateral mesh $\Sigma^{*}$ composed from one half of diagonals of $\Sigma$. (d) Those diagonals in $\Phi$ which are parallel to the previous ones according to Proposition 2 constitute a mesh $\Phi^{*}$ reciprocal-parallel to $\Sigma^{*}$ : vertices in $\Sigma^{*}$ correspondence faces of $\Phi^{*}$. (e) and (f) show the reciprocal-parallel mesh pair $\Sigma^{* *}, \Phi^{* *}$ composed from the diagonals omitted in (c) and (d)

The construction of a discrete Christoffel dual (i.e., $\Phi$ from $\Sigma$ ) for hexagonal meshes is easy, as there are enough degrees of freedom. The fact that $\operatorname{dim} \mathcal{P}(P)=4$ leads to a three-dimensional space of parallel polygons $Q$ with $\operatorname{area}(P, Q)=0$. Consequently adding a new hexagon of $\Phi$ to already constructed ones is an operation which has $3-k$ degrees of freedom, where $k$ is the number of known vertices (which are shared with already existing neighbors of the hexagon to be constructed).

Example Figure 13 shows a hexagonal mesh $\Sigma$ which is circumscribed to the unit sphere and its Christoffel dual mesh $\Phi$ which assumes the shape of an Enneper's surface. This example is constructed as follows: We start with an isothermic curvatureline parameterization $x(u, v)$ of the known smooth Enneper's surface $M$. Next we tile the parameter domain with nonconvex hexagons-we used dilates of the hexagon with vertices $(0,0),\left(\frac{1}{2}, \frac{1}{4}\right),(1,0),(1,1),\left(\frac{1}{2}, \frac{3}{4}\right),(0,1)$. Mapping the vertices of this tiling with the parameterization $x$ yields a hexagonal mesh $\Phi_{0}$ with nonplanar faces inscribed to $M$ (it looks very much like Fig. 13, right). Each planar hexagon has a center, say $\left(u^{*}, v^{*}\right)$, and we consider $x\left(u^{*}, v^{*}\right)$ as the center of the corresponding spatial hexagon. Next we construct the mesh $\Sigma$ by parallel translating the tangent planes of $M$ in the centers of hexagons such that they touch the unit sphere. Vertices $q_{i}$ of $\Sigma$ are found by intersection of planes (see Fig. 13, left). Having constructed $\Sigma$, 
(a): $\Sigma$

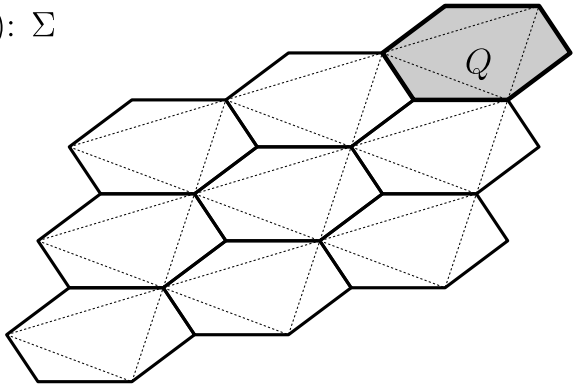

(c): $\Sigma^{*}$

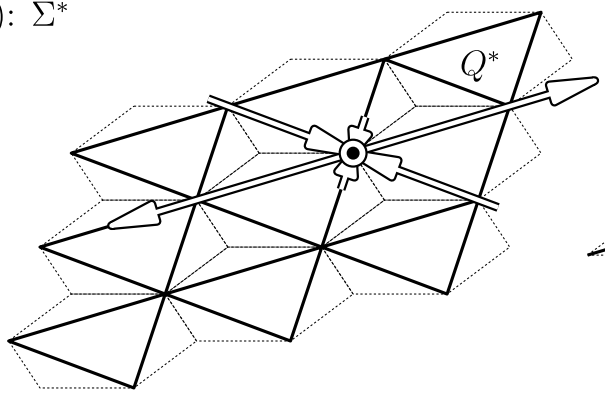

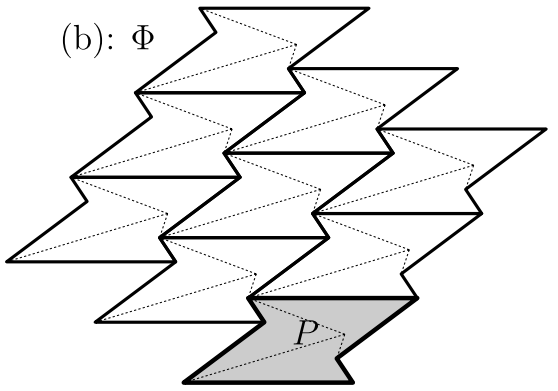

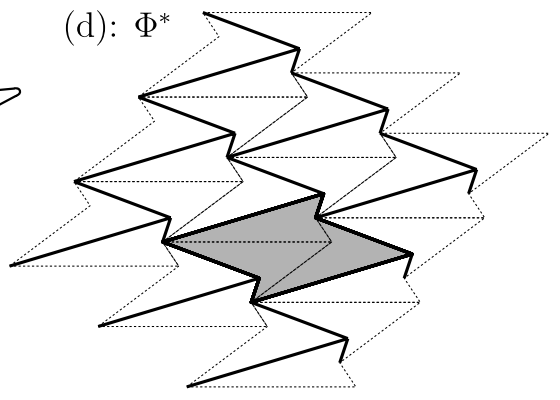

(e): $\Sigma^{* *}$

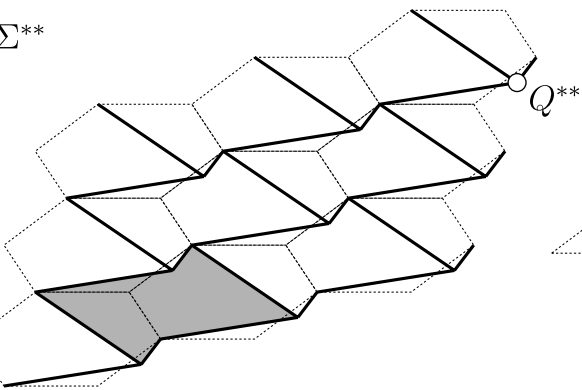

(f)

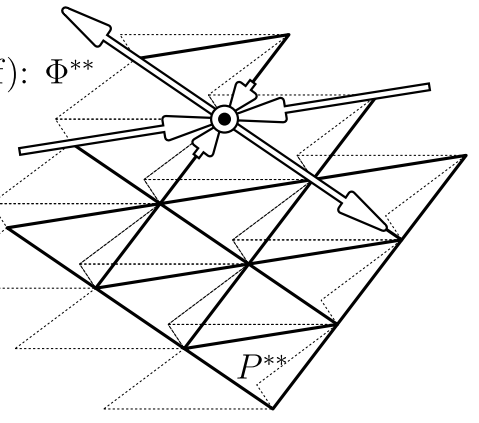

Fig. 16 Reciprocal-parallel meshes $\Sigma^{*}, \Phi^{*}$ derived from Christoffel dual meshes $\Sigma$, $\Phi$. The edges around a face in $\Phi^{*}$ serve as equilibrium forces acting in the edges adjacent to the corresponding vertex of $\Sigma^{*}$. (a) Hexagonal mesh $\Sigma$ with face $Q$. (b) Hexagonal mesh $\Phi$ of the same combinatorics such that corresponding faces $Q$ of $\Sigma$ and $P$ of $\Phi$ have vanishing mixed area. (c) Triangle mesh whose edges are derived polygons $Q^{*}$ in mesh $\Sigma$. (d) Hexagonal mesh whose edges are the lines $p_{1} p^{*}, p_{3} p^{*}, p_{5} p^{*}$ in each hexagon of $\Phi$, where $p^{*}$ is the degenerate polygon $P^{*}$. Note that $\Phi^{*}$ does not have planar faces. In (e) and (f), the geometric characterization of vanishing mixed area which yields diagonals and their parallels is read in reverse order compared to (a)-(d): Diagonals are taken from the nonconvex hexagons in the mesh $\Phi$

we find its Christoffel dual $\Phi$ by optimizing $\Phi_{0}$ so that mixed areas of corresponding faces $Q$ in $\Sigma$ and $P$ in $\Phi$ are zero and so that the corresponding edges $q_{i} q_{j}$ and $p_{i} p_{j}$ are parallel. This amounts to minimizing the quadratic functional

$$
\lambda_{\text {par }} \sum_{\text {edges }}\left\|\left(q_{i}-q_{j}\right) \times\left(p_{i}-p_{j}\right)\right\|^{2}+\lambda_{\text {mix }} \sum_{\text {faces }}\|\operatorname{area}(P, Q)\|^{2} .
$$


This is done by a standard conjugate gradient method and turns out not to change the shape of $\Phi_{0}$ much, despite the fact that the theoretical number of degrees of freedom is larger.

\subsection{Reciprocal Parallelity in Discrete Minimal Surfaces}

In the quad mesh case, there is an interesting connection of minimal surfaces with reciprocal-parallel meshes. A reciprocal-parallel mesh pair is defined as meshes which are combinatorial duals of each other (there is a correspondence face-vertex, vertex-face, and edge-edge) such that corresponding edges are parallel [8]. Proposition 2 immediately shows that $\Phi$ is a minimal surface with respect to $\Sigma$ if meshes composed from diagonals of quads in both $\Sigma, \Phi$ are reciprocally parallel [6]. This is illustrated in Fig. 15. It should be mentioned that reciprocal parallelity of meshes in connection with discrete minimal surfaces also occurs in other places, for instance, in [10].

For hexagonal meshes, we similarly can derive pairs of reciprocal-parallel meshes from a Christoffel dual pair $(\Sigma, \Phi)$. The geometric characterization of vanishing mixed area according to Theorem 7 and Fig. 7 leads to the configuration of lines in meshes $\Sigma$ and $\Phi$, which is illustrated in Figs. 16(a) and 16(b). Obviously, the configuration of diagonals in $\Sigma$ (Fig. 16(c)) is reciprocally parallel to the configuration of lines in $\Phi$, which consist of the to-be edges of degenerate derived polygons (Fig. 16(d)). A different choice of diagonals in $\Sigma$ would lead to a different pair of reciprocal-parallel meshes.

Obviously for each vertex of $\Sigma^{*}$, the cycle of edge vectors adjacent to the corresponding face of $\Phi^{*}$ serves as equilibrium forces acting on that vertex. Thus, $\Phi^{*}$ represents a collection of equilibrium forces for $\Sigma^{*}$. These forces (scaled) are shown by Fig. 16(c).

As the relation $\operatorname{area}(P, Q)=0$ is symmetric, the geometric configuration of the triangle $P^{*}$ and the degenerate triangle $Q^{*}$ exists also if the roles of $P$ and $Q$ (i.e., the roles of $\Sigma$ and $\Phi$ ) are exchanged: One half of the diagonals in $\Phi$ constitute a triangle mesh $\Phi^{* *}$ which possesses a reciprocal-parallel hexagonal mesh $\Sigma^{* *}$ associated with $\Sigma$ (see Figs. 16(e) and 16(f)). Figure 16(f) also shows equilibrium forces for $\Phi^{* *}$ which are a scaled version of the edges of $\Sigma^{* *}$.

\section{References}

1. Bobenko, A., Pinkall, U.: Discrete isothermic surfaces. J. Reine Angew. Math. 475, 187-208 (1996)

2. Bobenko, A., Suris, Y.: Discrete Differential Geometry: Integrable Structure. Graduate Studies in Mathematics, vol. 98. American Math. Soc., Providence (2008)

3. Bobenko, A.I., Suris, Y.: Discrete Koenigs nets and discrete isothermic surfaces. Int. Math. Res. Not. 2009, 1976-2012 (2009)

4. Bobenko, A., Hoffmann, T., Springborn, B.: Minimal surfaces from circle patterns: geometry from combinatorics. Ann. Math. 164, 231-264 (2006)

5. Pinkall, U., Polthier, K.: Computing discrete minimal surfaces and their conjugates. Exp. Math. 2, 15-36 (1993)

6. Pottmann, H., Liu, Y., Wallner, J., Bobenko, A., Wang, W.: Geometry of multi-layer free form structures for architecture. ACM Trans. Graph. 26(3), \#65 (2007) 
7. Pottmann, H., Wallner, J.: The focal geometry of circular and conical meshes. Adv. Comput. Math. 29, 249-268 (2008)

8. Sauer, R.: Differenzengeometrie. Springer, Berlin (1970)

9. Schneider, R.: Convex Bodies: The Brunn-Minkowski Theory. Cambridge University Press, Cambridge (1993)

10. Wallner, J., Pottmann, H.: Infinitesimally flexible meshes and discrete minimal surfaces. Monatshefte Math. 153, 347-365 (2008) 